3 Laitinen O, Leirisalo M, Skylv G. Relation between HLA-B27 and clinical features in patients with yersinia arthritis. Arthritis Rheum 1977; 20: 1121-4.

4 Leino R, Kalliomäki JL. Yersiniosis as an internal disease. Ann Intern Med 1974; 81: 458-61.

\section{Arthropathy of calcium pyrophosphate deposition disease and of haemochromatosis}

SIR, The recent report by Bourqui et al. ${ }^{1}$ documents again the rather specific structural joint damage seen in the hand and the wrist in patients with calcium pyrophosphate dihydrate (CPPD) crystal deposition disease. I would like to make several additional observations.

As noted in our previous article ${ }^{2}$ as well as in one by Martel et al., ${ }^{3}$ it is the distribution and the morphology of the articular abnormalities, especially in the hand and wrist, that are distinctive in radiographs in patients with CPPD crystal deposition disease. Although Bourqui et al. are indeed correct in their emphasis of the second and third metacarpophalangeal joints in this disease, their description of wrist alterations is incomplete. CPPD crystal deposition disease demonstrates a remarkable predilection for the radiocarpal joint in the wrist. Interosseous space narrowing between the distal radius and the scaphoid is most characteristic. Furthermore, the scaphoid moves proximally, leading to scalloped erosion of the distal radius, and the lunate migrates distally, with joint space narrowing mainly identified between it and the capitate. Separation, or dissociation, of the scaphoid and the lunate is subsequently observed, perhaps related to crystal deposition in the interosseous ligament.

At these articular locations as well as the metacarpophalangeal joints (and elsewhere) several morphological characteristics distinguish the arthropathy of CPPD crystal deposition disease from osteoarthrosis: (1) multiple (and often large) subchondral rarefactions or cysts; (2) irregularity or 'crumbling' of the articular surface; (3) severe progressive alterations that resemble those occurring in neuroarthropathy; (4) variable osteophyte formation; (5) single or multiple intra-articular osteocartilaginous bodies.

In their article Bourqui et al. acknowledge prior reports that indicate the similarity of the arthropathy of CPPD crystal deposition disease to that of haemochromatosis. We have recently compared the radiographic abnormalities in these two diseases ${ }^{4}$ and have recognised differences between the two. In haemochromatosis, as compared with CPPD crystal deposition disease, findings include more prevalent narrowing of the metacarpophalangeal joint spaces, including those in the fourth and fifth digits, peculiar hook-like osteophytes on the radial aspect of the metacarpal heads, and less prominent separation of the scaphoid and the lunate. These radiographic differences indicate that the arthropathy of haemochromatosis is related to factors additional to the presence of CPPD crystals.
Department of Radiology,

DONALD RESNICK

VA Medical Center,

3350 La Jolla Village Drive,

San Diego, CA 92161 .

USA

\section{References}

1 Bourqui M, Vischer T L, Stasse P, Docquier C, Fallet G H. Pyrophosphate arthropathy in the carpal and metacarpophalangeal joints. Ann Rheum Dis 1983; 42: 626-30.

2 Resnick D, Niwayama G, Goergen T G, et al. Clinical radiographic and pathologic abnormalities in calcium pyrophosphate dihydrate deposition disease (CPPD): pseudogout. Radiology 1977; 122: 1-15

3 Martel W, Champion C K, Thompson G R, Carter T L. A roentgenologically distinctive arthropathy in some patients with the pseudogout syndrome. AJR 1970; 109: 587-605.

4 Adamson R C III. Resnik C S. Guerra J Jr. et al. Hand and wrist arthropathies of hemochromatosis and calcium pyrophosphate deposition disease: distinct radiographic features. Radiology 1983; 147: 377-81.

\section{Arthritis and angioimmunoblastic lymphadenopathy}

SIR, Angioimmunoblastic lymphadenopathy is a lymphoproliferative condition of unknown aetiopathogenesis.' Typical features include lymphadenopathy, hepatosplenomegaly, cutaneous rash, pleuropulmonary lesions, and hypergammaglobulinaemia. Both arthralgia and arthritis have also been reported occasionally. ${ }^{2-4}$

A 49-year-old woman presented with fever, anorexia, weight loss, and symmetrical polyarthritis. These symptoms had been present for the last 11 months. There was no former history of viral infection or drug ingestion. Physical examination revealed a chronically ill patient with temperature of $39^{\circ} \mathrm{C}$. Numerous small, movable, rubbery and nontender lymph nodes were palpated in the cervical region. Synovial thickening, warmth, and tenderness of wrist, elbow, and knee joints were noted, as well as moderate bilateral knee effusion. The ESR was $100 \mathrm{~mm} / \mathrm{h}$. Protein electrophoresis showed a polyclonal hypergammaglobulinaemia. The latex test for rheumatoid factor and the antinuclear antibody test were negative.

Aspiration of the left knee revealed a clear fluid with a moderate number of leucocytes, $67 \%$ of which were polymorphonuclears. Synovial fluid protein, glucose, and C3 were within normal ranges. Culture was sterile and crystals were not observed. A radiograph of the left wrist showed periarticular osteoporosis and narrowing of the radiocarpal joint space with irregular subchondral erosions.

A lymph node biopsy was performed. Histological lesions consisted of diffuse effacement of nodal architecture with a pleomorphic cellular proliferation in which immunoblasts, plasma cells, histiocytes, and lymphocytes predominated. In addition, vascular proliferation-arborising blood vessels-and prominent eosinophilic PAS-positive interstitial material were seen. 